\title{
Effects of Sodium Sulfate and Surfactants on Papain Treatment of Wool Fabrics
}

\author{
In Young Kim ${ }^{\dagger}$ \\ Dept. of Clothing \& Textiles, Sookmyung Women's University; Seoul, Korea
}

\begin{abstract}
This study identifies the effects of sodium sulfate and surfactants in the papain treatment of wool fabrics using L-cysteine and EDTA as activators. The research method involves the use of $2 \%$ L-cysteine and 7\% EDTA as activators at optimal conditions, papain treatment of wool fabrics with the joint use of sodium sulfates and surfactants, and measurements of the weight loss rate, tensile strength, and whiteness. Results showed that for both $2 \%$ L-cysteine and $7 \%$ EDTA, the maximum papain activity appeared at $0.5 \%$ sodium sulfate concentration. In both cases, the papain activity was enhanced at sodium sulfate concentrations lower than $0.5 \%$. In contrast, the papain activity declined at sodium sulfate concentrations higher than $0.5 \%$. The joint use of EDTA with $0.5 \%$ sodium sulfate was proven to be very effective in improving the papain activity. The joint use of $2 \%$ L-cysteine with $0.5 \%$ sodium sulfate appeared slightly effective in improving the activity but resulted in excessive decrease in the tensile strength and whiteness, compared to improvement in the activity. The joint use of surfactants, in the case of L-cysteine, interrupted the papain activity and decreased the tensile strength regardless of the surfactant type and concentration. In the case of EDTA, however, the joint use with 0.1-5\% non-ionic surfactants, $0.1-0.5 \%$ anionic surfactants, and $0.1 \%$ cationic surfactant appeared to improve the papain activity. The maximum papain activity was observed when $0.1 \%$ of surfactant was used, regardless of the surfactant type. The nonionic surfactant was the most effective in improving the papain activity.
\end{abstract}

Key words : wool, papain, sodium sulfate, surfactant

\section{Introduction}

In the fiber industry, processing methods that use green-technology draw significant attention as environmental regulations are strengthened. One of the most noted processing methods is the use of enzymes. Novel approaches are being carried out to identify new enzymes and examine the enzymes that have been used for fiber processing. Accordingly, papain, a widely known vegetable protease, is studied multilaterally.

As a preceding study on papain, Song et al.(2009) reported that in wool/polyester blended fabric treatments using papain, the optimum activation conditions were $\mathrm{pH} 7.5,70^{\circ} \mathrm{C}, 10 \%$ papain, 60 minutes, with L-cysteine and sodium sulfite as activators. Under these conditions, the hydrophilic properties, whiteness, and dyeability increased. Song and Song(2008) proved that in wool/polyester blended fabric treatments using papain, the joint use of Lcysteine and sodium sulfite was more efficient for surface modifications than the individual use of each material. Lee et al.(2010) reported that in chitosan non-woven fabric treatment using papain, the amine bond was hydrolyzed but its internal structure did not change, and that the optimum activation conditions were $\mathrm{pH} 8$,

$†$ Corresponding author; In Young Kim

Tel. +82-53-810-2791, Fax. +82-53-810-4687

E-mail: inyoung@sm.ac.kr $60^{\circ} \mathrm{C}, 10 \%$ papain, and 60 minutes. Sung et al.(2008) reported that L-cysteine and EDTA were efficient as activators in the treatment of wool fiber with papain, and that the optimum activation conditions of $\mathrm{pH} 7.5,70^{\circ} \mathrm{C}, 5 \%$ papain, 30 minutes with $2 \%$ L-cysteine, and 180 minutes with 7\% EDTA led to a decreased contact angle, increased alkali solubility and dyeability. The aforementioned studies focused on the optimum conditions and activators for papain.

However, the activation of enzymes is affected by other factors such as surfactants, chelators, and reducing agents. In particular, surfactants facilitate the penetration and adsorption of the enzyme in addition to fiber swelling, which requires further investigations. Accordingly, previous studies regarding surfactants and other agents for enzyme processing were examined, and it was found that most studies focused on surfactants for cellulase and pectinase for cotton and cotton/blended fabrics(Kim \& Song, 2008; Li \& Hardin, 1998; Losonezi et al., 2004; Seo et al., 2009; Yoshimura et al., 2003; Yu et al., 2009), which poses the need for further investigations regarding surfactants and other agents for papain treatment of wool fiber.

This study identifies the effects of sodium sulfate and surfactants in the papain treatment of wool fabrics using L-cysteine and EDTA as activators and puts forth new data that can help in the commercialization of shrink-proof treatments of wool fabrics using papain. Because the joint use of sodium sulfateis expected to improves the papain solubility, and the joint use of surfactants is predicted to help 
the penetration and adsorption of enzyme and fiber swelling(Cavaco-Paul \& Gubitz, 2003; Glazer \& Smith, 1971).

The research method involves the use of $2 \%$ L-cysteine and $7 \%$ EDTA as activators at optimal conditions, papain treatment of wool fabrics with the joint use of sodium sulfates and surfactants, and measurements of the weight loss rate, tensile strength, and whiteness.

\section{Experiments}

\subsection{Materials}

The experimental fabric, $100 \%$ grey wool, was scoured in a shaking water bath (Jeio Tech, South Korea) containing 5\% sodium carbonate at liquor-to-fabric ratio of $1: 40$, at $40^{\circ} \mathrm{C}$. Subsequently, the mass was dried at room temperature and a $150 \mathrm{rpm}$ agitation speed was applied for 30 minutes. The characteristics of the experimental fabric are presented in Table 1.

The enzyme used was papain, a protease originating from Carica Papaya. The properties of papain are presented in Table 2.

One unit is the amount of papain that liberated $1 \mu \mathrm{M}$ of N-benzoyl-L-arginine ethyl ester per minute at $\mathrm{pH} 6.2,25^{\circ} \mathrm{C}$.

The buffer solution used was a mixture of Trizma ${ }^{\circledR} \mathrm{HCl}$ and Trizma ${ }^{\circledR}$ Base (Sigma Chemical Co., USA). The $\mathrm{pH}$ of the buffer solution was controlled with $1 \mathrm{M}$ hydrochloric acid and $1 \mathrm{M}$ sodium hydroxide (Duksan Pure Chemicals Co., South Korea). L-cysteine (Yakuri Pure Chemicals Co., Japan) and EDTA (Ducksan Pure Chemicals Co., South Korea) were used as activators. Tween 85 (Sigma Chemicals Co., USA) and Span 40 (Duksan Pure Chemicals Co., South Korea) were used as the non-ionic surfactants, LAS (Sodium Laurylbenzenesulfonate, Japan) as the anionic surfactant, and SnogenCat-800 (Daeyoung Chemicals Co., South Korea) as the cationic surfactant. The other reagents included sodium sulfate (Ducksan Pure Chemicals Co., South Korea). All the aforementioned reagents were first-class products.

\subsection{Experiment methods}

\subsubsection{Papain treatment}

The papain treatment was carried out using a shaking water bath

Table 1. Characteristics of the experimental fabric

\begin{tabular}{ccccc}
\hline Fiber (\%) & Weave & $\begin{array}{c}\text { Fabric Count } \\
\text { (yarns/inch) }\end{array}$ & $\begin{array}{c}\text { Fabric Weight } \\
\left(\mathrm{g} / \mathrm{m}^{2}\right)\end{array}$ & $\begin{array}{c}\text { Thickness } \\
(\mathrm{mm})\end{array}$ \\
\hline Wool 100 & Plain & $47 \times 43$ & 86.2 & 0.275 \\
\hline
\end{tabular}

Table 2. Properties of the enzyme papain

\begin{tabular}{ccccc}
\hline Enzyme & Source & Activity & Form & Manufacturer \\
\hline $\begin{array}{c}\text { Papain } \\
\text { (EC 3.4.22.2) }\end{array}$ & Carica papaya & 0.5 unit/mg & Powder & Fluka \\
\hline
\end{tabular}

(Jeio Tech., South Korea) under optimal activation conditions at the liquor-to-fabric ratio of 1:40, $\mathrm{pH} 7.5,5 \%$ (o.w.f.) papain, and $75^{\circ} \mathrm{C}$ for 30 minutes with $2 \% \mathrm{~L}$-cysteine and 7\% EDTA as activator. The buffer solution used was a mixture of $0.5 \mathrm{M}$ tris base and $0.5 \mathrm{M}$ tris $\mathrm{HCl}$. The aforementioned papain treatment conditions were the same as those in the paper of Sung and $\operatorname{Kim}(2008)$ reports. To investigate the effects of sodium sulfate on the treatment of wool fabrics using papain, the sodium sulfate concentration was changed to $0.1,0.5,1$, and $5 \%$ (o.w.f.) and the sodium sulfate was used in conjunction with the activators. For investigating the surfactant effects on the treatment of the wool fabric using papain, the concentrations of the anionic surfactant LAS, the cationic surfactant Snogen cat-800, and the non-ionic surfactants Tween 85 and Span 40 were changed to $0.1,0.5,1$, and $5 \%$ (o.w.f.), respectively, and they were used in conjunction with the activators. To inactivate the enzyme after the treatment, distilled water was used with the liquor-to-fabric ratio of $1: 40$ and at $90^{\circ} \mathrm{C}$ for 10 minutes.

\subsubsection{Measurement of weight loss rate}

The weight loss rate was calculated using the following formula after measuring the dried weights before and after the enzyme treatment and after the specimen was dried at $105-110^{\circ} \mathrm{C}$ for 90 minutes using a drier (Jeio Tech, South Korea) and conditioned in a desiccator for 30 minutes. The average results of five test runs are reported.

Weight loss $(\%)=\frac{W_{1}-W_{2}}{W_{1}} \times 100$

$\mathrm{W}_{1}$ : Fabric weight before the enzyme treatment

$\mathrm{W}_{2}$ : Fabric weight after the enzyme treatment

\subsubsection{Measurement of tensile strength}

The tensile strength was measured using a tensile strength tester (SS-121A, Sungshin Testing Machine Co., South Korea) in accordance with KS K0520 (Revelled Strip Method). The basic measurement conditions were set at $4 \mathrm{~cm}$ position distance and $30 \mathrm{~mm} /$ min for the tensile strength, and only the inclination directions were measured. The average results of five test runs are reported.

\subsubsection{Measurement of whiteness}

The whiteness was measured using a Computer Color Matching System $(\mathrm{J} \times 777$, Japan; hereinafter referred to as ' $\mathrm{CCM}$ '). The average results of five test runs are reported.

\section{Results and Discussion}

\subsection{Effects of sodium sulfate on papain activity}

Fig. 1-3 show the weight loss rate, tensile strength, and white- 


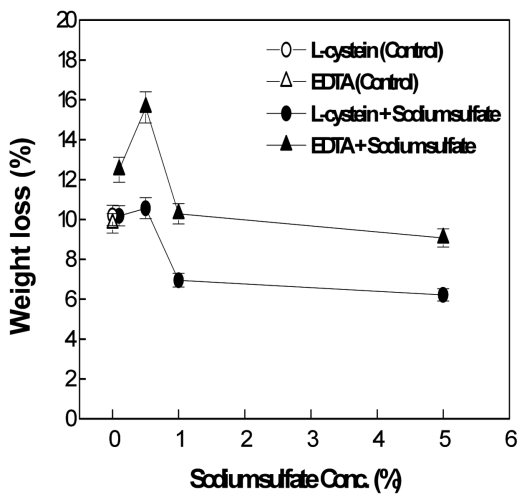

Fig. 1. Weight loss of wool fabrics treated by papain with the L-cysteine, EDTA andsodium sulfate.

ness corresponding to the sodium sulfate concentrations in the papain treatments of wool fabrics, using 2\% L-cysteine and $7 \%$ EDTA as activators, under optimal activation conditions.

As presented in Fig. 1, the weight loss observed when the activator $\mathrm{L}$-cysteine was used jointly with $1 \%$ sodium sulfate appeared similar to that observed for $0 \%$ sodium sulfate (referred to as the control hereinafter). Slightly increased weight loss was observed when L-cysteine was used jointly with $0.5 \%$ sodium sulfate. However, compared to the weight loss observed for the control, the corresponding values observed for 1 or $5 \%$ sodium sulfate with Lcysteine was significantly lower. In the case of EDTA as the activator, the weight loss observed when EDTA was jointly used with $0.5 \%$ sodium sulfate significantly increases up to the maximum weight loss of $15.62 \%$. The weight loss observed for EDTA jointly used with sodium sulfate in excess of $5 \%$, however, remained almost the same as those observed for the control. Therefore, the joint use of L-cysteine or EDTA with less than $0.5 \%$ of sodium sulfate increased the papain activity, but the joint use with sodium sulfate present in excess of $0.5 \%$ did not affect or even hinder the enzyme activity. This can be attributed to the fact that a small amount of neutral salt improves the papain solubility, but increasing neutral salt concentrations lowers the solubility of papain, leading to precipitation of papain in the solution(Glazer \& Smith, 1971). The result of Fig. 1 was different with the result of the previous study without sodium sulfate, that is, weight loss with EDTA islower than that with L-cysteine(Sung et al., 2010). This is because the joint use of sodium sulfate improves the role of EDTA as an activator but did not help the role of L-cysteine.

As shown in Fig. 2, in the both of 2\% L-cysteine and 7\% EDTA, the tensile strength decreased than that of the control by the joint use with all concentration of sodium sulfate. The tensile strength decreased to its minimum value with the joint use of either $2 \% \mathrm{~L}$ cysteine or $7 \%$ EDTA with $0.5 \%$ sodium sulfate. The joint use with

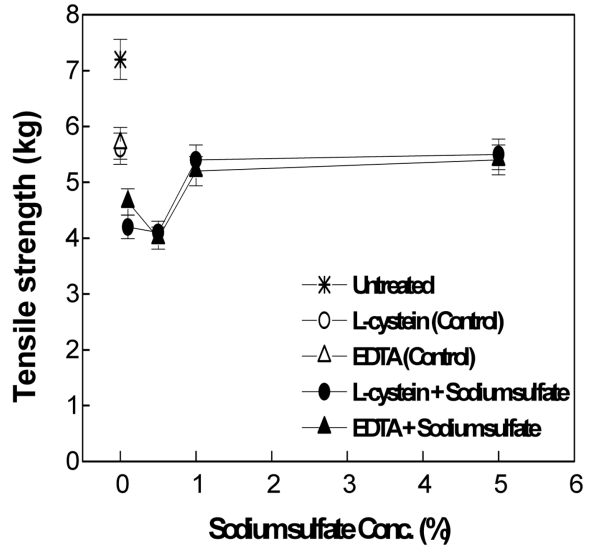

Fig. 2. Tensile strength of wool fabrics treated by papain with the Lcysteine, EDTA and sodium sulfate.

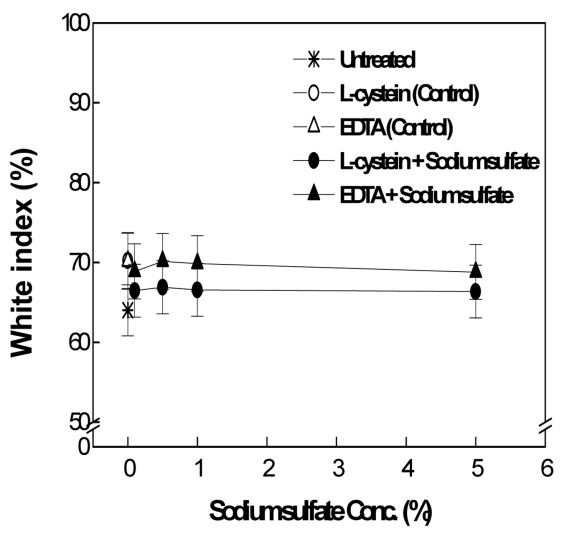

Fig. 3. White index of wool fabrics treated by papain with the Lcysteine, EDTA and sodium sulfate.

1 or $5 \%$ sodium sulfate presented strengths similar to those of the control. On comparing the test results of the tensile strength with the weight loss presented in Fig. 1, we can see that the joint use of 0.1 or $0.5 \%$ sodium sulfate leads to increased weight loss but decreased tensile strengths in both the $2 \%$ L-cysteine and $7 \%$ EDTA activators. On the other hand, with the joint use of 1 or $5 \%$ sodium sulfates, the tensile strength increased when $2 \%$ L-cysteine was used, while the weight loss rate decreased; however, when $7 \%$ EDTA was used, the tensile strength appeared similar to that of the control despite the decrease in weight loss.

Fig. 3 presents the whiteness measurement results. The whiteness for $2 \% \mathrm{~L}$-cysteine at $0.1 \%$ of sodium sulfate was lower than that for the control; however, no further decreases in whiteness were observed with increasing sodium sulfate concentration. With $7 \%$ EDTA, however, the whiteness at all concentrations of sodium sulfate appeared similar the control. 


\subsection{Effects of surfactants on papain activity}

While handling water-insoluble substrates in an enzyme treatment process, surfactants are used to improve the interactions between the enzyme and the substrates and to enhance the activity. Surfactants are divided into four kinds (cationic, anionic, zwitterionic, and nonionic), and the charged surfactants interact electrostatically with the charged amino acid chains present on a protein surface. However, the surfactants can also sometimes lead to the deactivation of the enzymes depending on the kinds of substrates and the origin of the enzymes. The suitability of the surfactant should thus be verified before its application(Cavaco-Paul \& Gubitz, 2003). Therefore, L-cysteine and EDTA were used as the activators to verify the surfactant effects in treating wool fabrics using papain under optimal vitalization conditions.

Fig. 4-9 present the measurement results of the weight loss, tensile strength, and whiteness in the papain treatments of wool fabrics under optimal vitalization conditions in the presence of different surfactant types (anionic surfactant LAS, non-ionic surfactants Tween 85 and Span 40, and cationic surfactant Snogen Cat-800) and concentrations.

As shown in Fig. 4, the weight loss observed for $2 \%$ L-cysteine used jointly with the surfactants was lower than that observed for the $0 \%$ surfactants (referred to as the control hereinafter) for all the four different surfactants used; the magnitude of the decrease in weight loss increased with surfactant concentration. This is attributed to enzyme deactivation at high surfactant concentration, as its secondary and tertiary structures denature due to surfactant adhesion on the enzyme(Yurika et al., 2003). As presented in Fig. 5, the weight loss observed for 7\% EDTA in conjunction with the nonionic surfactants Tween 85 or Span 40 was higher than the control (no surfactant) at all concentrations of the surfactant. The highest weight loss was observed at $0.1 \%$ surfactant and decreased as the concentration increased. For samples containing 7\% EDTA along with the anionic surfactant LAS, increased weight loss was observed at LAS concentrations lower than $1 \%$, when compared to the control, and decreased at concentrations of surfactant higher than $1 \%$. The highest rate was observed at $0.1 \%$ LAS, and the rate gradually decreased as the LAS concentration increased. When 7\% EDTA was jointly used with the cationic surfactant Snogen Cat800 , an increased weight loss rate, compared to that of the control, was observed only at the $0.1 \%$ concentration, and gradually decreased as the concentration increased. The decrease in enzyme activity at higher surfactant concentrations occurs because the enzyme activity is interrupted by the micelles formed by the surfactant at increasing surfactant concentration. The magnitude of weight loss varied with the surfactant type in the following order: non-ionic surfactants Tween 85 and Span $40>$ anionic surfactant

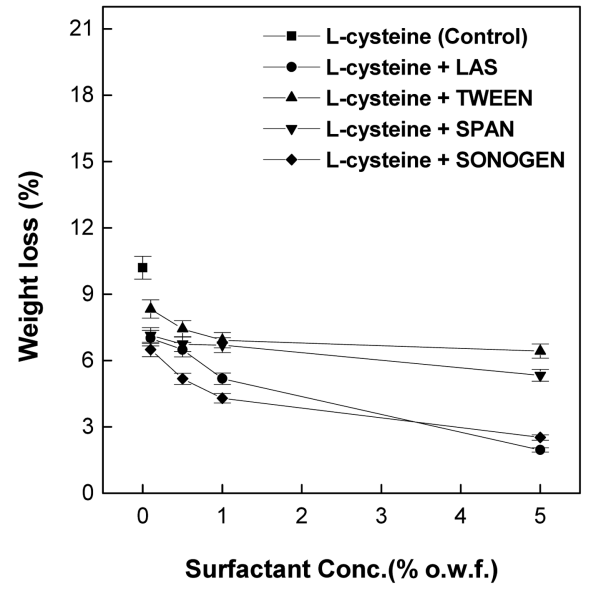

Fig. 4. Weight loss of wool fabrics treated by papain with the L-cysteine and surfactants.

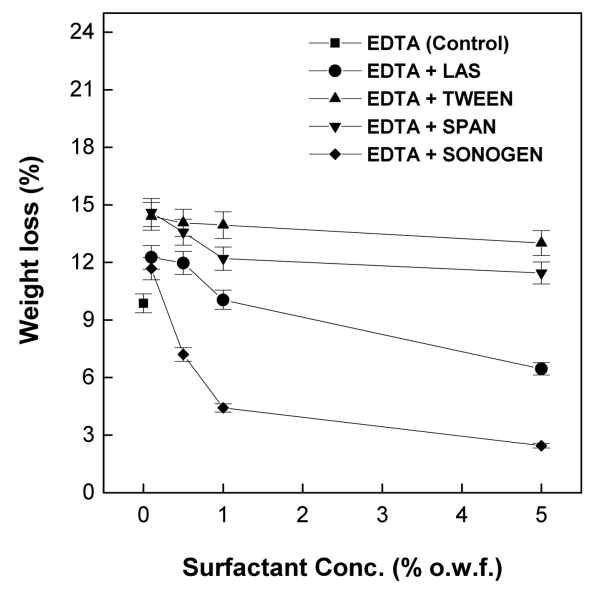

Fig. 5. Weight loss of wool fabrics treated by papain with the EDTA and surfactants.

LAS $>$ cationic surfactant Snogen Cat-800. It has been reported that the non-ionic surfactants have high enzyme suitability, whereas the anionic surfactants have lower suitability. According to previous studies(Yurika et al., 2003), the enzyme structure is broken and destroyed by the microenvironment constructed by the anionic surfactant SDS. On the other hand, the interruption of the enzyme activity caused by a cationic surfactant is because cationic surfactant is absorbed by the wool fabric and adheres to the negatively charged enzyme.

As presented in Fig. 6 and 7, the tensile strength observed when either 2\% L-cysteine or 7\% EDTA was jointly used the surfactant was mostly lower than that of the control. For all the surfactants tested, the lowest value of tensile strength was obtained at $0.1 \%$ of the surfactant; however, the tensile strength gradually increased 


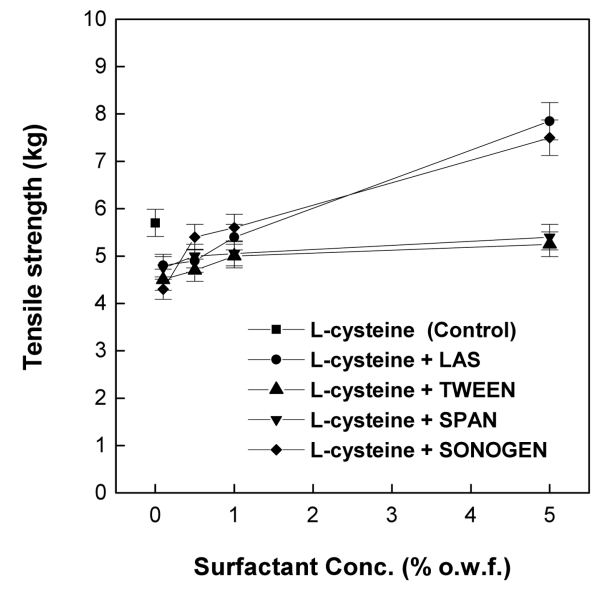

Fig. 6. Tensile strength of wool fabrics treated by papain with the Lcysteine and surfactants.

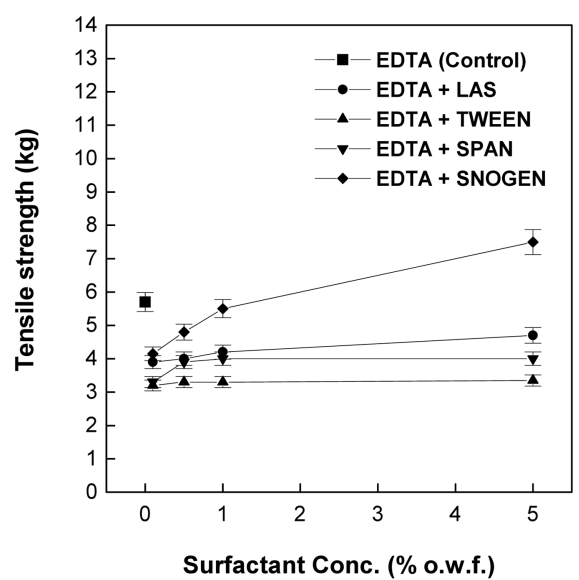

Fig. 7. Tensile strength of wool fabrics treated by papain with the EDTA and surfactant.

with further increase in the surfactant concentration. Compared with the trend in weight loss presented in Fig. 5, in the case of $2 \%$ L-cysteine, although the weight loss observed at all concentrations of all surfactants was lower than that of the control, the tensile strength was lower than that of the control. In the case of $7 \%$ EDTA, the tensile strengths observed in the presence of cationic surfactant concentrations higher than $0.5 \%$ and anionic surfactant concentration of only $5 \%$ was lower than that of the control although the weight loss was lower than that of the control. This is because papain was penetrated deep into the fibers by surfactants and hydrolyzed the endo-cuticle layers and cortexes of wool fibers(Na, 2001). Fig. 6 and 7 show that the increase in tensile strength depends on the surfactant type in the following order: nonionic surfactants Tween 85 and Span $40<$ anionic surfactant LAS $<$ cationic surfactant Snogen Cat-800.

As shown in Fig. 8 and 9, the whiteness observed for the control

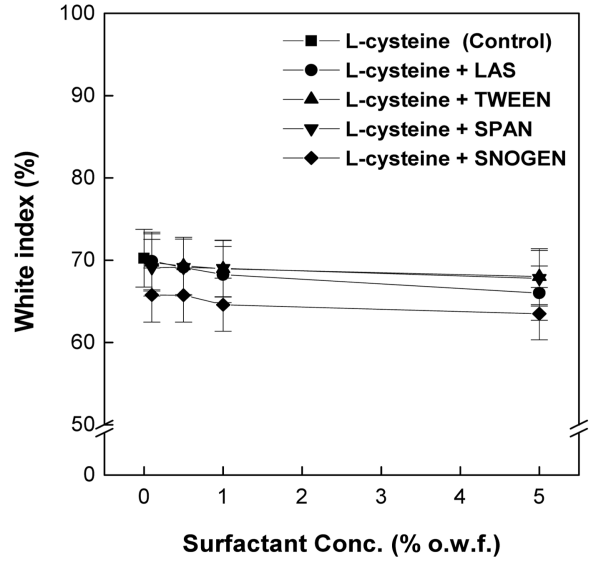

Fig. 8. Whiteness index of wool fabrics treated by papain with the Lcysteine and surfactants.

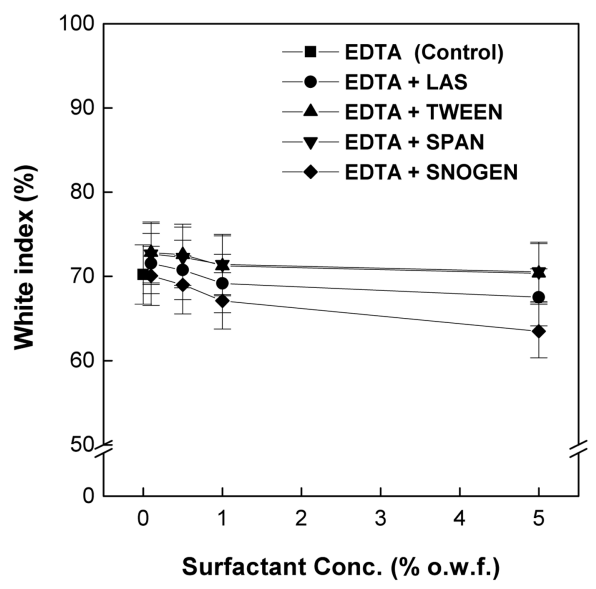

Fig. 9. Whiteness index of wool fabrics treated by papain with the EDTA and surfactants.

was similar to those observed for $2 \%$ L-cysteine used in conjunction with the nonionic surfactants Tween 85 and Span 40 and the anionic surfactant LAS at all concentrations. In contrast, the whiteness observed for $2 \%$ L-cysteine with the cationic surfactant Snogen Cat-800 decreased at all concentrations of the surfactant compared to the control. In the case of 7\% EDTA, similar or slightly higher whiteness values, compared to the control, were observed for all concentrations of the non-ionic surfactants Tween 85 and Span 40 and the anionic surfactant LAS. However, slightly decreased whiteness values were observed for all concentrations of the cationic surfactant Snogen Cat-800 in the presence of $7 \%$ EDTA. The maximum whiteness was observed for $0.1 \%$ for all the surfactants, which gradually decreased as the concentration increased. The results of the whiteness measurements correspond to the result of the weight loss measurements. Therefore, the whiteness measurement results also show that the surfactant effects 
papain activity. Previous studies reported that during the papain treatments of wool fabrics, the whiteness of the wool fabric increased as the papain reacted with the colored wool proteins, parts of which were hydrolyzed to water-soluble amino acids. Thus, the papain activity was enhanced(Na, 2001).

\section{Conclusion}

The effects of sodium sulfate and surfactants on the papain activity in the shrink-proof treatments of wool fabrics using papain are summarized below:

For both 2\% L-cysteine and 7\% EDTA, the maximum papain activity appeared at $0.5 \%$ sodium sulfate concentration. In both cases, the papain activity was enhanced at sodium sulfate concentrations lower than $0.5 \%$. In contrast, the papain activity declined at sodium sulfate concentrations higher than $0.5 \%$. The joint use of EDTA with $0.5 \%$ sodium sulfate was proven to be very effective in improving the papain activity. The joint use of $2 \%$ L-cysteine with $0.5 \%$ sodium sulfate appeared slightly effective in improving the activity but resulted in excessive decrease in the tensile strength and whiteness, compared to improvement in the activity. The joint use of surfactants, in the case of L-cysteine, interrupted the papain activity and decreased the tensile strength regardless of the surfactant type and concentration. In the case of EDTA, however, the joint use with $0.1-5 \%$ non-ionic surfactants, $0.1-0.5 \%$ anionic surfactants, and $0.1 \%$ cationic surfactant appeared to improve the papain activity. The maximum papain activity was observed when $0.1 \%$ of surfactant was used, regardless of the surfactant type. Among the various surfactant types, the non-ionic surfactant was proven to be the most effective in improving the papain activity.

\section{References}

Cavaco-Paulo, A., \& Gubitz, G. M. (2003). Textile processing with enzymes. Cambridge England;Woodhead publishing limited.

Glazer, A. N., \& Smith, E. L. (1971). Papain and other plant sulfhydryl proteolytic enzymes. The enzymes(3rded.), 3, 501-546.
Kim, H. R., \& Song, W. S. (2008). Effects of triton X-100 and calcium chloride on the porcine pancreas lipase treatment of PET fabrics. Journal of the Korean Society of Clothing and Textiles, 32(6), 911-8.

Lee, S. H., Kim, H. R., Lee, B. H., \& Song, W. S. (2010). Enzymatic hydrolysis of chitosan fiber using cellulase and papain. Journal of the Korean Fiber Society, 47(3), 212-221.

Li, Y., \& Hardin, I. R. (1998). Enzymatic scouring of cotton Surfactants, agitation, and selection of enzymes. Textile Chemist and Colorist, 30(9), 23-29.

Losonezi, A., Csiszar, E., \& Szakacs, G. (2004). Bleachability and dyeing properties of biopretreated and conventionally scoured cotton fabrics. Textile Research Journal, 74(6), 501-508.

$\mathrm{Na}$, Y. J. (2001). Bio-bleaching method and optimum conditions for wool blend fabrics. Journal of the Korean Fiber Society, 38(9), 445-452.

Song, H. J., Kim, H. R., \& Song, W. S. (2009). Dyeing properties and scouring of wool/polyester blend fabrics using papain from carica papaya. Journal of the Korean Society of Clothing and Textiles, 32(2), 213-221.

Song, H. J., \& Song, W. S. (2008). Effects of mixed activators on enzymatic activation for wool polyester blend fabrics. Journal of the Korean Society of Clothing and Textiles, 33(9), 1462-1466.

Na, Y. J., Seo, H. Y. Song, W. S., \& Kim, H. R. (2009). Enzymatic processing and property of denim by acid cellulase. Journal of the Korean Society for Clothing Industry, 11(3), 465-468.

Sung, J. M., \& Kim, I. Y. (2008). The effects of L-cysteine, EDTA in papain treatment of wool fabrics. Journalof the Korean Society of Dyers and Finishers, 20(2), 9-18.

Sung, J. M., Kim, I. Y., \& Song, W. S. (2010). Mechanical and dyeing properties of wool fabrics treated with papain. Journal of the Korean Society of Clothing and Textiles, 34(4), 697-702.

Yoshimura, Y. T., Abe, I., \& Sawada, K. (2003). Promoting effect of surfactants on enzyme treatment of wool. Sen'I Gakkaishi, 59(1), 35-29.

Yu, S. S., Kim, H. R., \& Song, W. S. (2009). Effects of surfactants on pectinase treatment of cotton/chitosan blends. Journal of the Korean Society of Clothing and Textiles, 33(12), 1965-1970.

Yurika, Y., Takeru, O., Ikuo, A., \& Kazuya, S. (2003). Promoting effect of surfactants on enzyme treatment of wool. Sen'I Gakkaishi, $59(1), 69-73$

(Received 10 March 2014; 1st Revised 18 March 2014; 2nd Revised 27 March 2014; Accepted 2 March 2014)

Copyright $(0) 2014$ (by) the authors. This article is an open access article distributed under the terms and conditions of the Creative Commons Attribution license (http://creativecommons.org/licenses/by/3.0/), which permits unrestricted use, distribution, and reproduction in any medium, provided the original work is properly cited. 